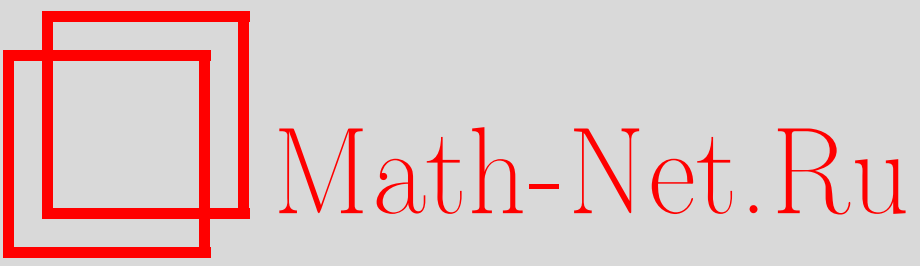

Б. Х. Сендов, Экстремальные задачи для алгебраических многочленов, УМН, 2005, том 60, выпуск 6, 175-186

DOI: https://doi.org/10.4213/rm1682

Использование Общероссийского математического портала Math-Net.Ru подразумевает, что вы прочитали и согласны с пользовательским соглашением

http://www.mathnet.ru/rus/agreement

Параметры загрузки:

IP : 3.81 .55 .215

26 апреля 2023 г., 16:19:09 


\section{ЭКСТРЕМАЛЬНЫЕ ЗАДАЧИ ДЛЯ АЛГЕБРАИЧЕСКИХ МНОГОЧЛЕНОВ}

Бл. СЕндов

Пусть $L(p)$ - линейный оператор на множестве нормализованных алгебраических многочленов $p(z)=\left(z_{1}-z\right)\left(z_{2}-z\right) \cdots\left(z_{n}-z\right)$ с $z_{1} z_{2} \cdots z_{n}=1$. Нас интересует значение

$$
[L]=\sup \left\{\min \left\{\left|L(p)\left(z_{k}\right)\right|: k=1,2, \ldots, n\right\}: z_{1} z_{2} \cdots z_{n}=1\right\}
$$

для различных линейных операторов. Наша мотивация состоит в том, что гипотеза Смейла о среднем значении может быть представлена в виде равенства $[L]=1-$ $1 /(n+1)$ для линейного оператора

$L(p)(z)=L\left(\sum_{k=0}^{n} a_{k} z^{k}\right)=\sum_{k=0}^{n} \frac{1}{k+1} a_{k} z^{k}=\frac{1}{z} \int_{0}^{z} p(u) d u, a_{0}=1, a_{n}=(-1)^{n}$.

Библиограффия: 7 названий.

\section{СОДЕРЖАНИЕ}

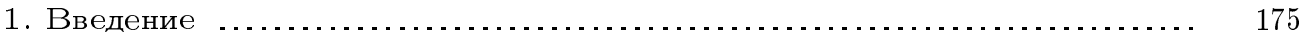

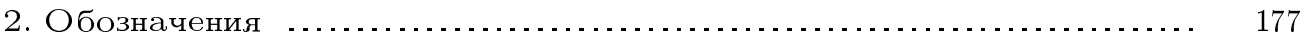

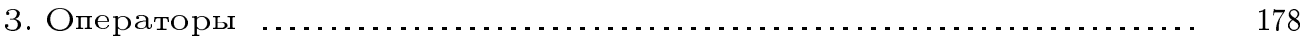

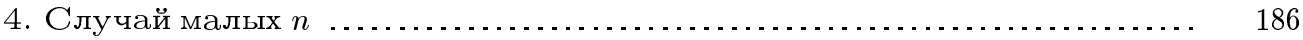

Список литературы ............................................ 186

\section{1. Введение}

С 1981 г. знаменитая гипотеза о среднем значении филдсовского медалиста Стефана Смейла [4] формулировалась с точки зрения численного метода последовательных приближений нулей многочлена $p$. При таком подходе было желательно найти наименьшую положительную постоянную $c$, удовлетворяющую условию

$$
\left|p^{\prime}(z)\right| \geqslant \frac{1}{c}\left|\frac{p(\zeta)-p(z)}{\zeta-z}\right|
$$

для по крайней мере одного нуля многочлена $p^{\prime}$ (критической точки $p$ ) и для всех $z$ в комплексной плоскости $\mathbb{C}$. Достаточно установить неравенство (1) только при $z=0$ 
и для всех многочленов $p$, удовлетворяюших условиям $p(0)=0$ и $p^{\prime}(0) \neq 0$. Смейл сформулировал следуюшую гипотезу о величине наименьшей постоянной $c$, известную как гипотеза Смейла о среднем значении.

ГИПОТеЗА 1. Пусть $p$ - многочлен степени $n$, причем $p(0)=0$ и $p^{\prime}(0) \neq 0$. Тогда

$$
\min \left\{\left|\frac{p(\zeta)}{\zeta p^{\prime}(0)}\right|: p(\zeta)=0\right\} \leqslant 1-\frac{1}{n} .
$$

Гипотеза 1 тривиальна при $n=2$ и доказана для $n=3,4$. Следуюший частный случай принадлежит Д. Тишлеру [5].

ТЕОРема 1. Гипотеза 1 выполняется для многочлена р, если все критические точки многочлена р имеют равные модули.

В качестве ослабления гипотезы 1 можно разрешить постоянной $с$ быть больше 1. С. Смейл проверил утверждение $(2)$ для $c=4$. А. $\Phi$. Бердон, Д. Минда и Т. В. Нг [1] доказали неравенство (2) для

$$
c=\alpha_{n}=4^{(n-2) /(n-1)},
$$

а Г. Шмайссер [2] привел простое доказательство неравенства (2) для

$$
c=\beta_{n}=\frac{2^{n}-(n+1)}{n(n-1)} .
$$

Асимптотически величина $\beta_{n}$ много больше $\alpha_{n}$, но при $2 \leqslant n \leqslant 7$ имеем $\beta_{n}<\alpha_{n}$.

Тишлер [5], [6] сфформулировал следуюшую более сильную гипотезу.

ГИПОТЕЗА 2. Пусть $p$ - многочлен степени $n$, и пусть $p(0)=0 u p^{\prime}(0) \neq 0$. Тогда

$$
\min \left\{\left|\frac{1}{2}-\frac{p(\zeta)}{\zeta p^{\prime}(0)}\right|: p(\zeta)=0\right\} \leqslant \frac{1}{2}-\frac{1}{n}
$$

Эта более общая гипотеза была доказана Тишлером при $n=3,4$. Недавно Дж. Тайсон [7] опубликовал статью, в которой наиболее интересен следуюший результат.

ТеОРема 2. Для любого $n \geqslant 5$ существует многочлен р степени $n$ с $p(0)=0$ и $p^{\prime}(0) \neq 0$, удовлетворяющий условию

$$
\min \left\{\left|\frac{1}{2}-\frac{p(\zeta)}{\zeta p^{\prime}(0)}\right|: p(\zeta)=0\right\}>\frac{1}{2}-\frac{1}{n}
$$

Иначе говоря, Тайсон доказал, что гипотеза 2 неверна при $n>4$.

Цель настояшей статьи - сформулировать задачу о минимаксе для симметрических многочленов, включающую гипотезы 1 и 2. 


\section{2. Обозначения}

Пусть $\mathscr{S}_{n}$ - множество нормализованных алгебраических многочленов

$$
\begin{aligned}
p(z) & =\left(z_{1}-z\right)\left(z_{2}-z\right) \cdots\left(z_{n}-z\right) \\
& =\sum_{m=0}^{n}(-1)^{m} S_{n, n-m}\left(z_{1}, z_{2}, \ldots, z_{n}\right) z^{m}=\sum_{m=0}^{n} a_{m} z^{m}
\end{aligned}
$$

с $z_{1} z_{2} \cdots z_{n}=1$, где

$$
S_{n, m}\left(z_{1}, z_{2}, \ldots, z_{n}\right), \quad m=0,1,2, \ldots, n,
$$

- элементарные симметрические функции степени $m$.

Пусть $U(p)$ - оператор на множестве алгебраических многочленов $p \in \mathscr{S}_{n}$. Положим

$$
\begin{aligned}
\|U(p)\| & =\min \left\{\left|U(p)\left(z_{k}\right)\right|: k=1,2, \ldots, n\right\}, \\
{[U] } & =\sup \left\{\|U(p)\|: p \in \mathscr{S}_{n}\right\} .
\end{aligned}
$$

Многочлен $p^{*} \in \mathscr{S}_{n}$ назьвается әкстремальнылм для оператора $U$, если $[U]=$ $\left\|U\left(p^{*}\right)\right\|$.

Рассмотрим класс линейных операторов $L$ на множестве алгебраических многочленов следуюшего вида:

$$
L(p)(z)=\sum_{m=0}^{n} \alpha_{m} a_{m} z^{m},
$$

где $\alpha_{0}, \alpha_{1}, \ldots, \alpha_{n}$ - вешественные числа. Тогда

$$
\begin{aligned}
L(p)\left(z_{1}\right) & =\sum_{m=0}^{n}(-1)^{m} \alpha_{m} S_{n, n-m}\left(z_{1}, z_{2}, \ldots, z_{n}\right) z_{1}^{m} \\
& =\sum_{m=0}^{n-1}(-1)^{m} \lambda_{m+1} S_{n-1, n-m-1}\left(z_{2}, z_{3}, \ldots, z_{n}\right) z_{1}^{m+1},
\end{aligned}
$$

где

$$
\lambda_{k}=\alpha_{k-1}-\alpha_{k}, \quad k=1,2, \ldots, n .
$$

Положим

$$
\Lambda=\left\{\lambda_{1}, \lambda_{2}, \ldots, \lambda_{n}\right\}
$$

и отождествим оператор $L=L_{\Lambda}$ с вектором $\Lambda$.

ОПРЕДЕЛЕНИЕ 1 . Обозначим через $\mathscr{L}_{n}$ множество всех линейных операторов $L_{\Lambda}$, отвечаюших $n$-мерным векторам $\Lambda$ с неотрицательными координатами.

Очевидно, что $\mathscr{L}_{n}-$ замкнутьй вьпукльй конус.

Изучение операторов из конуса $\mathscr{L}_{n}$ мотивируется тем, что гипотеза Смейла [4] отвечает оператору $L_{\Lambda} \in \mathscr{L}_{n}$ с вектором

$$
\Lambda=\left\{\frac{1}{1 \cdot 2}, \frac{1}{2 \cdot 3}, \ldots, \frac{1}{n(n+1)}\right\} .
$$

Заметим, что гипотеза 2 соответствует оператору $L_{\Lambda} \in \mathscr{L}_{n}$ с

$$
\Lambda=\left\{0, \frac{1}{2 \cdot 3}, \ldots, \frac{1}{n(n+1)}\right\}
$$

и, в нашей формулировке, $n-1$ заменено на $n$. 


\section{3. Операторы}

Начнем с простого утверждения.

ЛЕмма 1. Для любого оператора $L_{\Lambda} \in \mathscr{L}_{n}$ значение $\left[L_{\Lambda}\right]$ конечно и выполняется неравенство

$$
\left[L_{\Lambda}\right] \leqslant \sum_{m=0}^{n-1}\left(\begin{array}{c}
n-1 \\
m
\end{array}\right) \lambda_{m+1}
$$

ДокАЗАТЕЛЬСТво. Из равенства $z_{1} z_{2} \cdots z_{n}=1$ следует, что

$$
S_{n-1, n-m-1}\left(z_{2}, z_{3}, \ldots, z_{n}\right)=\frac{1}{z_{1}} S_{n-1, m}\left(\frac{1}{z_{2}}, \frac{1}{z_{3}}, \ldots, \frac{1}{z_{n}}\right), \quad m=0,1,2, \ldots, n-1 .
$$

Пусть нули занумерованы так, что $\left|z_{1}\right| \leqslant\left|z_{2}\right| \leqslant \cdots \leqslant\left|z_{n}\right|$. Тогда

$$
\begin{aligned}
{\left[L_{\Lambda}\right] } & \leqslant\left|L_{\Lambda}(p)\left(z_{1}\right)\right|=\left|\sum_{m=0}^{n-1}(-1)^{m} \lambda_{m+1} S_{n-1, m}\left(\frac{z_{1}}{z_{2}}, \frac{z_{1}}{z_{3}}, \ldots, \frac{z_{1}}{z_{n}}\right)\right| \\
& \leqslant \sum_{m=0}^{n-1}\left(\begin{array}{c}
n-1 \\
m
\end{array}\right) \lambda_{m+1} .
\end{aligned}
$$

ПРЕДЛОЖЕНИЕ 1. Равенство

$$
\left[c L_{\Lambda}\right]=|c|\left[L_{\Lambda}\right]
$$

справедливо для любого $\Lambda$ и любой постоянной $с$, причем

$$
\left[L_{\Lambda}\right]=0 \text { тогда и только тогда, когда } \Lambda=I=\{0,0, \ldots, 0\} \text {. }
$$

Положим

$$
\begin{gathered}
\zeta_{k}=e^{2(k-1) \pi i / n}, \quad k=1,2, \ldots, n, \\
\theta_{n}(z)=1+(-z)^{n}=\prod_{k=1}^{n}\left(\zeta_{k}-z\right)
\end{gathered}
$$

И

$$
\kappa_{n}(z)=(1-z)^{n}
$$

Очевидно, что $\theta_{n}(z)$ и $\kappa_{n}(z)$ - многочлены, принадлежашие множеству $\mathscr{S}_{n}$.

ПРЕДЛОЖЕНИЕ 2. Если $p^{*}(z)$ - экстремальный многочлен для оператора $L_{\Lambda} \in$ $\mathscr{L}_{n}$, то и многочлен

$$
p_{m}^{*}(z)=p^{*}\left(\zeta_{m} z\right), \quad m=1,2, \ldots, n,
$$

экстремальньц для $L_{\Lambda}$.

Доказательство следует из того факта, что нули $p_{m}^{*}-$ это точки $z_{k} \zeta_{m}^{-1}$, где $k=$ $1,2, \ldots, n$, и $L_{\Lambda}\left(p^{*}\right)\left(z_{k}\right)=L_{\Lambda}\left(p_{m}^{*}\right)\left(z_{k} \zeta_{m}^{-1}\right)$. 
ПРЕДЛОЖЕНИЕ 3. Для любого $L_{\Lambda} \in \mathscr{L}_{n}$ имеем

$$
L_{\Lambda}\left(\theta_{n}\right)\left(\zeta_{k}\right)=\lambda_{1}+\lambda_{2}+\cdots+\lambda_{n}, \quad k=1,2, \ldots, n
$$

$$
L_{\Lambda}\left(\kappa_{n}\right)\left(\zeta_{k}\right)=\sum_{m=0}^{n-1}(-1)^{m}\left(\begin{array}{c}
n-1 \\
m
\end{array}\right) \lambda_{m+1}, \quad k=1,2, \ldots, n
$$

ОПРЕДЕЛЕНИЕ 2 . Обозначим через $\mathscr{L}\left(\theta_{n}\right)$ множество операторов $L_{\Lambda} \in \mathscr{L}_{n}$, для которых многочлен $\theta_{n}$ является экстремальным.

Множество операторов $L_{\Lambda} \in \mathscr{L}_{n}$, для которых многочлен $\kappa_{n}$ является экстремальным, мы обозначим символом $\mathscr{L}_{+}\left(\kappa_{n}\right)$, если

$$
\sum_{m=0}^{n-1}(-1)^{m}\left(\begin{array}{c}
n-1 \\
m
\end{array}\right) \lambda_{m+1} \geqslant 0
$$

и символом $\mathscr{L}_{-}\left(\kappa_{n}\right)$, если

$$
\sum_{m=0}^{n-1}(-1)^{m}\left(\begin{array}{c}
n-1 \\
m
\end{array}\right) \lambda_{m+1} \leqslant 0 .
$$

Если $L_{\Lambda} \in \mathscr{L}\left(\theta_{n}\right)$, то мы будем также говорить, что $\Lambda \in \mathscr{L}\left(\theta_{n}\right)$, и то же относится к $\mathscr{L}_{+}\left(\kappa_{n}\right)$ и $\mathscr{L}_{-}\left(\kappa_{n}\right)$.

Лемма 2. Множества $\mathscr{L}\left(\theta_{n}\right), \mathscr{L}_{+}\left(\kappa_{n}\right)$ и $\mathscr{L}_{-}\left(\kappa_{n}\right)$ - замкнутые выпуклье конусы.

ДокАЗАТЕЛЬСтво. Нам нужно доказать, что если $L_{\Lambda}, L_{\Omega} \in \mathscr{L}\left(\theta_{n}\right)$ и $\alpha>0, \beta>0$ вьполняется соотношение $L_{\Gamma}=\alpha L_{\Lambda}+\beta L_{\Omega} \in \mathscr{L}\left(\theta_{n}\right)$. Предположим противное. Пусть $\theta_{n}-$ не экстремальный многочлен для $L_{\Gamma}$. Тогда

$$
\left[L_{\Gamma}\right]>\alpha\left(\sum_{m=1}^{n} \lambda_{m}\right)+\beta\left(\sum_{m=1}^{n} \omega_{m}\right)
$$

Пусть $g$ - экстремальньй многочлен для $L_{\Gamma}$. Тогда по предложению 3 имеем

$$
\begin{aligned}
\left\|L_{\Gamma}(g)\right\| & =\min \left\{\left|L_{\Gamma}(g)(\zeta)\right|: g(\zeta)=0\right\} \\
& =\min \left\{\left|\alpha L_{\Lambda}(g)(\zeta)+\beta L_{\Omega}(g)(\zeta)\right|: g(\zeta)=0\right\} \\
& \leqslant \min \left\{\left|\alpha L_{\Lambda}\left(\theta_{n}\right)(\zeta)+\beta L_{\Omega}\left(\theta_{n}\right)(\zeta)\right|: \theta_{n}(\zeta)=0\right\} \\
& =\alpha\left(\sum_{m=1}^{n} \lambda_{m}\right)+\beta\left(\sum_{m=1}^{n} \omega_{m}\right),
\end{aligned}
$$

что противоречит формуле (8).

Тот факт, что $\mathscr{L}_{+}\left(\kappa_{n}\right)$ и $\mathscr{L}_{-}\left(\kappa_{n}\right)$ - выпуклые конусы, можно доказать аналогично. 
Заметим, что гипотеза 1 эквивалентна соотношению

$$
\left\{\frac{1}{1 \cdot 2}, \frac{1}{2 \cdot 3}, \ldots, \frac{1}{n(n+1)}\right\} \in \mathscr{L}\left(\theta_{n}\right)
$$

а гипотеза 2 - соотношению

$$
\left\{0, \frac{1}{2 \cdot 3}, \ldots, \frac{1}{n(n+1)}\right\} \in \mathscr{L}\left(\theta_{n}\right)
$$

Хорошо известно, что любой элемент замкнутого вьпуклого конуса в конечномерном евклидовом пространстве может быть представлен в виде неотрицательной линейной комбинации неприводимых элементов этого конуса. (Элемент конуса назьвается неприводимьм, если это - периферический элемент, не являющийся суммой двух различных элементов этого конуса.) Неприводимые элементы конуса $\mathscr{L}_{n}$ - это векторы

$$
e_{n, m}=\left\{\lambda_{1}, \lambda_{2}, \ldots, \lambda_{n}\right\}, \quad m=1,2, \ldots, n,
$$

где $\lambda_{m}=1$ и $\lambda_{l}=0$ при $l \neq m$. Очевидно, любой элемент $\mathscr{L}_{n}$ есть неотрицательная линейная комбинация векторов $(9)$.

Из леммы 1 и предложения 3 вытекает следуюший результат.

ПРЕДЛОЖЕНИЕ 4. Векторь $e_{n, 1} u e_{n, n}$ принадлежат конусу $\mathscr{L}\left(\theta_{n}\right)$. Вектор $e_{n, m}$ принадлежст конусу $\mathscr{L}_{+}\left(\kappa_{n}\right)$, если $m$ четно, и конусу $\mathscr{L}_{-}\left(\kappa_{n}\right)$, если т нечетно. Векторы еп,т неприводимы в любом содержащем их подконусе конуca $\mathscr{L}_{n}$

При $n \geqslant 3$ векторь $e_{n, m}$ не принадлежат $\mathscr{L}\left(\theta_{n}\right)$ при $m=2,3, \ldots, n-1$.

3.1. Операторы с двумя последовательными ненулевыми координатами. Мы ищем простые векторы, принадлежащие $\mathscr{L}\left(\theta_{n}\right)$.

ОПРЕДЕЛЕНИЕ 3. ПолОжим

$$
l_{n, m}(\alpha)=\left\{\lambda_{1}, \lambda_{2}, \ldots, \lambda_{n}\right\}, \quad \alpha \in[0,1]
$$

где $\lambda_{m}=1-\alpha, \lambda_{m+1}=\alpha$ и $\lambda_{l}=0$ при $n \neq m, m+1 ; m=1,3, \ldots, n-1$.

Из предложения 3 следует, что

$$
\left\|L_{l_{n, m}(\alpha)}\left(\theta_{n}\right)\right\|=\lambda_{m-1}+\lambda_{m}=1
$$

и

$$
\left\|L_{l_{n, m}(\alpha)}\left(\kappa_{n}\right)\right\|=\left|\left(\begin{array}{c}
n \\
m
\end{array}\right) \alpha-\left(\begin{array}{c}
n-1 \\
m-1
\end{array}\right)\right| .
$$

Поэтому справедливо 
ПРЕДЛОЖЕНИЕ 5 . Вектор $l_{n, m}(\alpha)$ не принадлежит конусу $\mathscr{L}\left(\theta_{n}\right)$, если $\alpha$ не принадлежит отрезку

$$
\left[\frac{m}{n}-\left(\begin{array}{c}
n \\
m
\end{array}\right)^{-1}, \frac{m}{n}+\left(\begin{array}{c}
n \\
m
\end{array}\right)^{-1}\right]
$$

Доказательство следует из неравенства

$$
\left\|L_{l_{n, m}(\alpha)}\left(\theta_{n}\right)\right\|<\left\|L_{l_{n, m}(\alpha)}\left(\kappa_{n}\right)\right\|,
$$

которое вьполняется, если $\alpha$ не принадлежит отрезку (10).

Возникает естественный вопрос: принадлежит ли вектор $l_{n, m}(\alpha)$ конусу $\mathscr{L}\left(\theta_{n}\right)$, если $\alpha$ принадлежит отрезку (10)? Ответ положителен при $m=1$ и $m=n-1$, но отрицателен в обшем случае, см. лемму 5 и предложение 6 .

Для доказательства леммы 5 нам нужно следующее утверждение.

ЛЕмма 3. Пусть $x_{1}, x_{2}, \ldots, x_{n}$ - положительные вещественные числа, $x_{1} x_{2} \cdots x_{n}=1$. Тогдa

$$
x_{1}+x_{2}+\cdots+x_{n} \leqslant x_{1}^{-n+1}+x_{2}^{-n+1}+\cdots+x_{n}^{-n+1} .
$$

ДокАЗАТЕЛЬСТво. Лемма тривиальна при $n=2$. При $n=3$ она доказана в [3]. Доказательство следует из очевидных неравенств

$$
x_{1}^{2}\left(x_{2}-x_{3}\right)^{2}+x_{2}^{2}\left(x_{3}-x_{1}\right)^{2}+x_{3}^{2}\left(x_{1}-x_{2}\right)^{2} \geqslant 0 .
$$

Действительно, при $x_{1} x_{2} x_{3}=1$ имеем

$$
\begin{aligned}
& x_{1}^{2}\left(x_{2}-x_{3}\right)^{2}+x_{2}^{2}\left(x_{3}-x_{1}\right)^{2}+x_{3}^{2}\left(x_{1}-x_{2}\right)^{2} \\
& \quad=2\left(x_{1}^{2} x_{2}^{2}+x_{2}^{2} x_{3}^{2}+x_{3}^{2} x_{1}^{2}-x_{1}^{2} x_{2} x_{3}-x_{2}^{2} x_{3} x_{1}-x_{3}^{2} x_{1} x_{2}\right) \\
& \quad=2\left(x_{1}^{-2}+x_{2}^{-2}+x_{3}^{-2}-x_{1}-x_{2}-x_{3}\right) \geqslant 0 .
\end{aligned}
$$

При $n \geqslant 4$ положим

$$
\begin{aligned}
f\left(x_{1}, x_{2}, \ldots, x_{n-1}\right)= & \left(x_{1} x_{2} \cdots x_{n-1}\right)^{n-1}+x_{1}^{-n+1}+x_{2}^{-n+1}+\cdots+x_{n-1}^{-n+1} \\
& -\left(x_{1} x_{2} \cdots x_{n-1}\right)^{-1}-x_{1}-x_{2}-\cdots-x_{n-1} .
\end{aligned}
$$

Соотношение

$$
\frac{\partial f}{\partial x_{k}}=0, \quad k=1,2, \ldots, n-1,
$$

является необходимым условием минимума $f$. Из (11) следует, что не сушествует таких трех индексов $k, l, s$, что

$$
x_{k} \neq x_{l} \neq x_{s} \neq x_{k} .
$$

Предположим противное. Пусть (12) вьполняется. Тогда из

$$
\frac{\partial f}{\partial x_{k}}=\frac{n-1}{x_{k}}\left(x_{1} x_{2} \cdots x_{n-1}\right)^{n-1}-(n-1) x_{k}^{-n}+\frac{1}{x_{k}}\left(x_{1} x_{2} \cdots x_{n-1}\right)^{-1}-1=0
$$


следует, что

$$
\begin{aligned}
& \frac{\partial f}{\partial x_{k}}-\frac{\partial f}{\partial x_{l}}=\left(\frac{1}{x_{k}}-\frac{1}{x_{l}}\right)\left[(n-1)\left(x_{1} x_{2} \cdots x_{n-1}\right)^{n-1}\right. \\
& \left.\quad-(n-1)\left(x_{k}^{-n+1}+x_{k}^{-n+2} x_{l}^{-1}+\cdots+x_{l}^{-n+1}\right)+\left(x_{1} x_{2} \cdots x_{n-1}\right)^{-1}\right]=0, \\
& \frac{\partial f}{\partial x_{k}}-\frac{\partial f}{\partial x_{s}}=\left(\frac{1}{x_{k}}-\frac{1}{x_{s}}\right)\left[(n-1)\left(x_{1} x_{2} \cdots x_{n-1}\right)^{n-1}\right. \\
& \left.\quad-(n-1)\left(x_{k}^{-n+1}+x_{k}^{-n+2} x_{s}^{-1}+\cdots+x_{s}^{-n+1}\right)+\left(x_{1} x_{2} \cdots x_{n-1}\right)^{-1}\right]=0
\end{aligned}
$$

и

$$
x_{k}^{-n+1}+x_{k}^{-n+2} x_{l}^{-1}+\cdots+x_{l}^{-n+1}=x_{k}^{-n+1}+x_{k}^{-n+2} x_{s}^{-1}+\cdots+x_{s}^{-n+1},
$$

или $x_{l}=x_{s}$, что противоречит (12).

$\mathrm{K}$ настоящему моменту мы доказали, что из (11) следует, что

$$
x_{1}=x_{2}=\cdots=x_{k}=u, \quad x_{k+1}=x_{k+2}=\cdots=x_{n-1}=v
$$

и

$$
\begin{aligned}
& f\left(x_{1}, x_{2}, \ldots, x_{n-1}\right)=g(u, v)=\left(u^{k} v^{n-k-1}\right)^{n-1} \\
& \quad+k u^{-n+1}+(n-k-1) v^{-n+1}-\left(u^{k} v^{n-k-1}\right)^{-1}-k u-(n-k-1) v .
\end{aligned}
$$

Поэтому из соотношения

$$
\frac{\partial g}{\partial u}=\frac{\partial g}{\partial v}=0
$$

следует, что $u=v$, и поэтому

$$
g(u, v)=h(u)=u^{(n-1)^{2}}+(n-2) u^{-n+1}-(n-1) u .
$$

Отсюда получаем

$$
\frac{\partial h}{\partial u}=\frac{n-1}{u^{n}}\left((n-1) u^{n^{2}-2 n}-u^{n}-n+2\right)=0
$$

и, наконец, $u=1$. Докажем, что из (11) следует соотношение

$$
\min \left\{f\left(x_{1}, x_{2}, \ldots, x_{n}\right): x_{k}>0\right\}=f(1,1, \ldots, 1)=0
$$


ЛЕмма 4. Для $z_{1}$ положим

$$
A_{1}=(1-\alpha) S_{n-1, n-m}\left(z_{2}, z_{3}, \ldots, z_{n}\right) z_{1}^{m}-\alpha S_{n-1, n-m-1}\left(z_{2}, z_{3}, \ldots, z_{n}\right) z_{1}^{m+1}
$$

и аналогично введем $A_{2}, A_{3}, \ldots, A_{n}$ для $z_{2}, z_{3}, \ldots, z_{n}$ соответственно. Пусть

$$
Z_{1}^{\prime}=S_{n-1, n-m}\left(z_{2}, z_{3}, \ldots, z_{n}\right), \quad Z_{1}^{\prime \prime}=z_{1} S_{n-1, n-m-1}\left(z_{2}, z_{3}, \ldots, z_{n}\right)
$$

при $z_{1}$, и аналогично введем $Z_{2}^{\prime}, Z_{2}^{\prime \prime}, \ldots, Z_{n}^{\prime}, Z_{n}^{\prime \prime}$ при $z_{2}, z_{3}, \ldots, z_{n}$ соответственно. Если

$$
M=S_{n, n-m}\left(z_{1}, z_{2}, \ldots, z_{n}\right)
$$

mo

$$
\sum_{k=1}^{n}\left|z_{k}\right|^{-2 m}\left|A_{k}\right|^{2}=\sum_{k=1}^{n}\left|Z_{k}^{\prime}-\alpha M\right|^{2}=\sum_{k=1}^{n}\left|Z_{k}^{\prime}\right|^{2}-\alpha(2 m-n \alpha)|M|^{2}
$$

$u$

$$
\sum_{k=1}^{n}\left|z_{k}\right|^{-2 m}\left|A_{k}\right|^{2}=\sum_{k=1}^{n}\left|Z_{k}^{\prime \prime}-(1-\alpha) M\right|^{2}=\sum_{k=1}^{n}\left|Z_{k}^{\prime \prime}\right|^{2}-(1-\alpha)(n-2 m+n \alpha)|M|^{2}
$$

ДокАЗАТЕЛЬСтво. Заметим, что

$$
Z_{1}^{\prime}+Z_{2}^{\prime}+\cdots+Z_{n}^{\prime}=m M \text { и } Z_{1}^{\prime \prime}+Z_{2}^{\prime \prime}+\cdots+Z_{n}^{\prime \prime}=(n-m) M .
$$

Тогда

$$
\begin{aligned}
\sum_{k=1}^{n}\left|z_{k}\right|^{-2 m}\left|A_{k}\right|^{2} & =\sum_{k=1}^{n}\left(Z_{k}^{\prime}-\alpha M\right)\left(\overline{Z_{k}^{\prime}}-\alpha \bar{M}\right) \\
& =\sum_{k=1}^{n}\left|Z_{k}^{\prime}\right|^{2}+n \alpha^{2}|M|^{2}-2 m \alpha|M|^{2}
\end{aligned}
$$

Формулу (14) можно доказать аналогично.

Мы теперь можем доказать следующее утверждение.

Лемма 5. Вектор $l_{n, 1}(\alpha)$ лежит в конусе $\mathscr{L}\left(\theta_{n}\right)$ при $\alpha \in[0,2 / n]$, а вектор $l_{n, n-1}(\alpha)$ принадлежит конусу $\mathscr{L}\left(\theta_{n}\right)$ при $\alpha \in[1-2 / n, 1]$.

ДОКАЗАТЕЛЬСТВО. При $m=1$ и $\alpha \in[0,2 / n]$ из (13) следует, что

$$
\sum_{k=1}^{n}\left|z_{k}\right|^{-2}\left|A_{k}\right|^{2} \leqslant \sum_{k=1}^{n}\left|Z_{k}^{\prime}\right|^{2}, \quad Z_{k}^{\prime}=\frac{z_{1} z_{2} \cdots z_{n}}{z_{k}}
$$

Согласно последнему неравенству, хотя бы для одного из чисел $k \in\{1,2, \ldots, n\}$, скажем, для $k=1$, имеем

$$
\left|z_{1}\right|^{-2}\left|A_{1}\right|^{2} \leqslant\left|Z_{1}\right|^{2}
$$


или

$$
\left|A_{1}\right|^{2} \leqslant\left|z_{1}\right|^{2}\left|Z_{1}^{\prime}\right|^{2}=\left|z_{1} z_{2} \cdots z_{n}\right|^{2}=1 \text {. }
$$

Для доказательства второй части леммы нужна лемма 3 . При $m=n-1$ и $\alpha \in$ $[1-2 / n, 1]$ имеем

$$
\sum_{k=1}^{n}\left|z_{k}\right|^{-2 n+2}\left|A_{k}\right|^{2} \leqslant \sum_{k=1}^{n}\left|Z_{k}^{\prime \prime}\right|^{2}=\sum_{k=1}^{n}\left|z_{k}\right|^{2} \leqslant \sum_{k=1}^{n}\left|z_{k}\right|^{-2 n+2} .
$$

Согласно последнему неравенству, хотя бы для одного из чисел $k \in\{1,2, \ldots, n\}$, скажем, при $k=1$, имеем

$$
\left|z_{1}\right|^{-2 n+2}\left|A_{1}\right|^{2} \leqslant\left|z_{1}\right|^{-2 n+2}
$$

или

$$
\left|A_{1}\right|^{2} \leqslant 1
$$

ПрЕДЛОЖЕНИЕ 6 . Вектор $l_{4,2}(\alpha)$ не принадлежит конусу $\mathscr{L}\left(\theta_{4}\right)$ при $\alpha \neq 1 / 2$. ДокАЗАТЕльство. Для $z_{1}$ положим

$$
A_{1}=(1-\alpha)\left(z_{2} z_{3}+z_{3} z_{4}+z_{4} z_{2}\right) z_{1}^{2}-\alpha\left(z_{2}+z_{3}+z_{4}\right) z_{1}^{3}
$$

и аналогично введем $A_{2}, A_{3}, A_{4}$ при $z_{2}, z_{3}, z_{4}$, соответственно. Пусть

$$
z_{1}=z_{2}=e^{i \varphi}, \quad z_{3}=z_{4}=e^{-i \varphi}, \quad z_{1} z_{2} z_{3} z_{4}=1 .
$$

Тогда

$$
A_{1}(\varphi)=1-\alpha+2(1-2 \alpha) e^{2 i \varphi}-\alpha e^{4 i \varphi} .
$$

Очевидно

$$
A_{2}(\varphi)=A_{1}(\varphi), \quad A_{3}(\varphi)=A_{4}(\varphi)=\overline{A_{1}(\varphi)},
$$

или

$$
\left|A_{1}(\varphi)\right|=\left|A_{2}(\varphi)\right|=\left|A_{3}(\varphi)\right|=\left|A_{4}(\varphi)\right|
$$

и

$$
\begin{gathered}
\min \left\{\left|A_{1}(\varphi)\right|,\left|A_{2}(\varphi)\right|,\left|A_{3}(\varphi)\right|,\left|A_{4}(\varphi)\right|\right\}=\left|A_{1}(\varphi)\right| . \\
\left|A_{1}(\varphi)\right|^{2}=A_{1}(\varphi) \overline{A_{1}(\varphi)}=5-18 \alpha+18 \alpha^{2}+4(1-2 \alpha)^{2} \cos 2 \varphi-2 \alpha(1-\alpha) \cos 4 \varphi .
\end{gathered}
$$

Величина $\left|A_{1}(\varphi)\right|^{2}$ достигает максимума для

$$
\cos 2 \varphi^{*}=\frac{(1-2 \alpha)^{2}}{2 \alpha(1-\alpha)}
$$

и

$$
\left|A_{1}\left(\varphi^{*}\right)\right|^{2}=1+4(1-2 \alpha)^{2}+\frac{(1-2 \alpha)^{2}}{\alpha(1-\alpha)} .
$$

Заметим, что

$$
0 \leqslant \frac{(1-2 \alpha)^{2}}{2 \alpha(1-\alpha)} \leqslant \frac{1}{4} \quad \text { для } \alpha \in[1 / 3,2 / 3] .
$$

Из (15) следует, что $\{0,1-\alpha, \alpha, 0\}$ может принадлежать $\mathscr{L}\left(\theta_{4}\right)$ при $\alpha=1 / 2$.

На основании предложений 5 и 6 получаем следующую гипотезу. 
ГипотезА 3. Векторы $l_{n, m}(m / n)$ принадлежат $\mathscr{L}\left(\theta_{n}\right)$ nри $m=2,3, \ldots, n-2$.

Лемма 6. Гипотеза 3 влечет гипотезу Смейла о среднем значении.

ДокАЗАтЕльСТво. Пусть гипотеза 3 справедлива. Тогда следующие векторы принадлежат $\mathscr{L}\left(\theta_{n}\right)$ :

$$
\begin{aligned}
& V_{1}=\{1,0,0,0, \ldots, 0,0,0\}, \\
& V_{2}=\{n-1,1,0,0, \ldots, 0,0,0\}, \\
& V_{3}=\{0, n-2,2,0, \ldots, 0,0,0\} \text {, } \\
& V_{n-1}=\{0,0,0,0, \ldots, 2, n-2,0\}, \\
& V_{n}=\{0,0,0,0, \ldots, 0,1, n-1\} .
\end{aligned}
$$

Простые вычисления показывают, что

$$
\left\{\frac{1}{1 \cdot 2}, \frac{1}{2 \cdot 3}, \ldots, \frac{1}{n(n+1)}\right\}=\frac{1}{n+1} V_{1}+\frac{1}{n+1} \sum_{k=2}^{n} \frac{1}{(k-1) k} V_{k}
$$

Заметим, что вектор

$$
\left\{0, \frac{1}{2 \cdot 3}, \ldots, \frac{1}{n(n+1)}\right\}
$$

не является положительной линейной комбинацией векторов $V_{1}, V_{2}, V_{3}, \ldots, V_{n}$ при $n \geqslant 4$. Этот факт не дает нового доказательства теоремы Тайсона (теоремы 2), но такое доказательство может быть получено нашими операторными методами.

В [3] получено решение задачи, обратной гипотезе 1.

Tеорема 3. Если $\Lambda=\{1,1,1, \ldots, 1\}$, mo $\left[L_{\Lambda}\right]=n u\{1,1,1, \ldots, 1\} \in \mathscr{L}\left(\theta_{n}\right)$.

Теорема 3 эквивалентна соотношению

$$
\sup \left\{\min \left\{\left|\zeta p^{\prime}(\zeta)\right|: p(\zeta)=0\right\}: p \in \mathscr{S}_{n}\right\}=n
$$

Его доказательство основано на тождестве

$$
\sum_{k=1}^{n} \frac{1}{z_{k} p^{\prime}\left(z_{k}\right)}=(-1)^{n-1}
$$

при $p \in \mathscr{S}_{n}$ с простыми нулями $z_{1}, z_{2}, \ldots, z_{n}$.

Заметим, что

$$
\{1,1,1, \ldots, 1\}=\frac{1}{n-1} \sum_{k=2}^{n} V_{k} .
$$




\section{4. Случай мальх $n$}

В этом разделе мы описьваем конусы $\mathscr{L}\left(\theta_{2}\right)$ и $\mathscr{L}\left(\theta_{3}\right)$.

4.1. Конус $\mathscr{L}\left(\theta_{2}\right)$. Из предложения 4 следует, что $\mathscr{L}\left(\theta_{2}\right)=\mathscr{L}_{2}$, и для любого $\Lambda=\left\{\lambda_{1}, \lambda_{2}\right\} \in \mathscr{L}_{2}$ имеем $[\Lambda]=\lambda_{1}+\lambda_{2}$. Доказательства гипотез 1 и 2 при $n=3$ следуют из последнего равенства.

4.2. Конус $\mathscr{L}\left(\theta_{3}\right)$. Из предложения 4 и леммы 5 получаем:

TEOPEMA 4. Beкmopbl

$$
U_{1}=\{1,0,0\}, \quad U_{2}=\{1,2,0\}, \quad U_{3}=\{0,2,1\} \quad u \quad U_{4}=\{0,0,1\}
$$

и коллинеарные им - это все неприводимые векторы в $\mathscr{L}\left(\theta_{3}\right)$.

Доказательства гипотез 1 и 2 при $n=4$ следуют из теоремы 4 поскольку

$$
\left\{\frac{1}{1 \cdot 2}, \frac{1}{2 \cdot 3}, \frac{1}{3 \cdot 4)}\right\}=\frac{1}{2} U_{1}+\frac{1}{12} U_{3}
$$

и

$$
\left\{0, \frac{1}{2 \cdot 3}, \frac{1}{3 \cdot 4}\right\}=\frac{1}{12} U_{3} .
$$

4.3. Конус $\mathscr{L}\left(\theta_{4}\right)$. Мы уже доказали, что неприводимые векторы в $\mathscr{L}\left(\theta_{3}\right)$ имеют вид

$$
V_{1}=\{1,0,0,0\}, \quad V_{2}=\{1,1,0,0\}, \quad V_{4}=\{0,0,1,1\} \quad \text { и } V_{5}=\{0,0,0,1\} .
$$

Для доказательства гипотезы Смейла о среднем значении при $n=5$ нужно показать, что

$$
V_{3}=\{0,1,1,0\} \in \mathscr{L}\left(\theta_{4}\right)
$$

\section{СПИСОК ЛИТЕРАТУРЫ}

[1] A.F. Beardon, D. Minda, T. W. Ng. Smale's mean value conjecture and the hyperbolic metric // Math. Ann. 2002. V. 322. №4. P. 623-632.

[2] Q. I. Rahman, G. Schmeisser. Analytic Theory of Polynomials. Oxford: Oxford Univ. Press, Clarendon Press, 2002.

[3] Bl. Sendov, N. Nikolov. Min problems for complex polynomials // East J. Approx. 2003. V. 9. № 4. P. 427-442.

[4] S. Smale. The fundamental theorem of algebra and complexity theory // Bull. Amer. Math. Soc. 1981. V. 4. № 1. P. 1-36.

[5] D. Tischler. Critical points and values of complex ploynomials // J. Complexity. 1989. V. 5. №4. P. 438-456.

[6] D. Tischler. Perturbations of critical fixed points of analytic mapes // Astérisque. 1994. V. 222. P. 407-422.

[7] J. Tyson. Conterexamples to Tischler's strong form of Smale's mean value conjecture // Bull. London Math. Soc. 2005. V. 37. № 1. P. 95-106. 